Revista de Matemática: Teoría y Aplicaciones 1999 6(2) : 87-96

CIMPA - UCR - CCSS ISSN: 1409-2433

\title{
RELATIONS OF K-TH DERIVATIVE OF DIRAC DELTA IN HYPERCONE WITH ULTRAHYPERBOLIC OPERATOR
}

\author{
Manuel A. Aguirre T.*
}

Received: December 3, 1998

\begin{abstract}
In this paper we prove that the generalized functions $\delta^{(k)}\left(P_{+}\right)-\delta^{(k)}(P), \delta^{(k)}\left(P_{-}\right)-$ $\delta^{(k)}(-P)$ and $\delta_{1}^{(k)}(P)-\delta_{2}^{(k)}(P)$ are concentrated in the vertex of the cone $P=0$ and we find their relationship with the ultrahyperbolic operator iterated $\left(k+1-\frac{n}{2}\right)$ times under condition $k \geq \frac{n}{2}-1$.
\end{abstract}

Keywords: distributions, generalized functions, distributions spaces, properties of distributions.

\section{Resumen}

En este trabajo se prueba que las funciones generalizadas $\delta^{(k)}\left(P_{+}\right)-\delta^{(k)}(P)$, $\delta^{(k)}\left(P_{-}\right)-\delta^{(k)}(-P)$ y $\delta_{1}^{(k)}(P)-\delta_{2}^{(k)}(P)$ están concentradas en el vértice del cono $P=0$ y encontramos sus relaciones con el operador ultrahiperbólico iterado $\left(k+1-\frac{n}{2}\right)$ veces bajo la condición $k \geq \frac{n}{2}-1$.

Palabras clave: distribuciones, functiones generalizadas, espacios de distribuciones, propiedades de distribuciones.

AMS Subject Classification: 46F.

\section{Introduction}

Let $x=\left(x_{1}, x_{2}, \cdots, x_{n}\right)$ be a point of the $\mathrm{n}$-dimensional Euclidean space $\mathrm{R}^{n}$.

Consider a quadratic form in $\mathrm{n}$ variables defined by

$$
P=P(x)=x_{1}^{2}+\ldots+x_{p}^{2}-x_{p+1}^{2}-\ldots-x_{p+q}^{2}
$$

* Núcleo Consolidado de Matemática Pura y Aplicada, Facultad de Ciencias Exactas-UNcentro, Pinto 399-C.P. (7000)-Tandil-Provincia de Buenos Aires, Argentina. 
where $p+q=n$ is the dimension of the space.

We call $\varphi(x)$ the $\mathrm{C}^{\infty}$ functions with compact support defined from $\mathbb{R}^{n}$ to $\mathbb{R}([2]$,page 4).

From [1], page 253, formula (2), the distribution $P_{+}^{\lambda}$ is defined by

$$
\left(P_{+}^{\lambda}, \varphi\right)=\int_{P>0}(P(x))^{\lambda} \varphi(x) d x
$$

where $\lambda$ is a complex number and $d x=d x_{1} d x_{2} \ldots d x_{n}$. For $\operatorname{Real}(\lambda) \geq 0$, this integral converges and is analytic function of $\lambda$. Analytic continuation to $\operatorname{Real}(\lambda)<0$ can be used to extend the definition of $\left(P_{+}^{\lambda}, \varphi\right)$. Further from [1], page 254, we have,

$$
\left(P_{+}^{\lambda}, \varphi\right)=\int_{0}^{\infty} u_{q}^{\lambda+\frac{p+q}{2}-1} \Phi_{\lambda}(u) d u
$$

where

$$
\begin{aligned}
{ }_{q} \Phi_{\lambda}(u) & =\frac{1}{4} \int_{0}^{\infty} t^{\frac{q-2}{2}}(1-t)^{\lambda} \phi_{1}(u, t u) d t \\
\phi(r, s) & =\phi_{1}(u, v) \\
\phi(r, s) & =\int \varphi d \Omega_{p} d \Omega_{q} \\
r & =\sqrt[2]{x_{1}^{2}+\cdots+x_{p}^{2}} \\
s & =\sqrt[2]{x_{p+1}^{2}+\ldots x_{p+q}^{2}}
\end{aligned}
$$

$d \Omega_{p}$ and $d \Omega_{q}$ are elements of surface are on the unit sphere in $\mathbb{R}^{p}$ and $\mathbb{R}^{q}$ respectively.

Similarly we can also defined the generalized $\mathrm{P}_{-}^{\lambda}$ by

$$
\left(P_{-}^{\lambda}, \varphi\right)=\int_{-P>0}(-P(x))^{\lambda} \varphi(x) d x
$$

Further we obtain

$$
\left(P_{-}^{\lambda}, \varphi\right)=\int_{0}^{\infty} v_{p}^{\lambda+\frac{p+q}{2}-1} \Phi_{\lambda}(v) d v
$$

where

$$
{ }_{p} \Phi_{\lambda}(u)=\frac{1}{4} \int_{0}^{\infty} t^{\frac{p-2}{2}}(1-t)^{\lambda} \phi_{1}(v t, v) d t
$$

From (1) the $P=0$ hypersurface is a hypercone with a singular point (the vertex) at the origin.

On the other hand, from [1], page 249, we have,

$$
\left(\delta^{(k)}(P), \varphi\right)=\int_{0}^{\infty}\left[\left(\frac{\partial}{2 s \partial s}\right)^{k}\left\{s^{q-2} \frac{\phi(r, s)}{2}\right\}\right]_{s=r} r^{p-1} d r
$$


and

$$
\left(\delta^{(k)}(P), \varphi\right)=(-1)^{k} \int_{0}^{\infty}\left[\left(\frac{\partial}{2 r \partial r}\right)^{k}\left\{r^{p-2} \frac{\phi(r, s)}{2}\right\}\right]_{r=s} s^{q-1} d s
$$

where $\phi(r, s)$ is defined by the equation (6).

Also from [1], page 250, the generalized functions $\delta_{1}^{(k)}(P)$ and $\delta_{2}^{(k)}(P)$ are defined by

$$
\left(\delta_{1}^{(k)}(P), \varphi\right)=\int_{0}^{\infty}\left[\left(\frac{\partial}{2 s \partial s}\right)^{k}\left\{s^{q-2} \frac{\phi(r, s)}{2}\right\}\right]_{s=r} r^{p-1} d r
$$

and

$$
\left(\delta_{2}^{(k)}(P), \varphi\right)=(-1)^{k} \int_{0}^{\infty}\left[\left(\frac{\partial}{2 r \partial r}\right)^{k}\left\{r^{p-2} \frac{\phi(r, s)}{2}\right\}\right]_{r=s} s^{q-1} d s
$$

where $\phi(r, s)$ is $r^{1-p} s^{1-q}$ multiplied by the integral of $\varphi$ over the surface $x_{1}^{2}+x_{2}^{2}+\cdots x_{p}^{2}=r^{2}$ and $x_{p+1}^{2}+x_{p+2}^{2}+\cdots x_{p+q}^{2}=s^{2}$.

The integrals converges and coincide for

$$
k<\frac{p+q-2}{2} .
$$

If, on the other hand,

$$
k \geq \frac{p+q-2}{2}
$$

these integrals must be understood in the sen se of their regularization (see [1], page 250).

Now in general $\delta_{1}^{(k)}(P)$ and $\delta_{2}^{(k)}(P)$ may not be the same generalized function.

Note that the definition of these generalized functions implies that in any case

$$
\delta_{2}^{(k)}(P)=(-1)^{k} \delta_{1}^{(k)}(-P) .
$$

From [1], page 278, the following formulae are valid,

$$
\left.\delta^{(k)}\left(P_{+}\right)=(-1)^{k} k ! \mathcal{R}\right\rceil s_{\lambda=-k-1} P_{+}^{\lambda}
$$

and

$$
\left.\delta^{(k)}\left(P_{-}\right)=(-1)^{k} k ! \mathcal{R}\right\rceil s_{\lambda=-k-1} P_{-}^{\lambda} .
$$

On the other hand, from [1], page 278, for odd $n$, as well as for even $\mathrm{n}$ and $k<\frac{n}{2}-1$ we have,

$$
\delta^{(k)}\left(P_{+}\right)=\delta_{1}^{(k)}(P)=\delta^{(k)}(P)
$$

and

$$
\delta^{(k)}\left(P_{-}\right)=\delta_{1}^{(k)}(-P) .
$$

While in the case of even dimension and $k \geq \frac{n}{2}-1$

$$
\delta^{(k)}\left(P_{+}\right)-\delta_{1}^{(k)}(P)
$$


and

$$
\delta^{(k)}\left(P_{-}\right)-\delta_{1}^{(k)}(-P)
$$

are generalized functions concentrated at the vertex of the $P=0$ cone ([1],page 279).

From [1], page 279 we have:

If $p$ and $q$ are both even and if $k \geq \frac{n}{2}-1$, then

$$
(-1)^{k} \delta^{(k)}\left(P_{+}\right)-\delta^{(k)}\left(P_{-}\right)=a_{q, n, k} L^{k+1-\frac{n}{2}}\{\delta(x)\}
$$

while in all other cases

$$
\delta^{(k)}\left(P_{-}\right)=(-1)^{k} \delta^{(k)}\left(P_{+}\right)
$$

$\operatorname{In}(25)$

$$
a_{q, n, k}=\frac{(-1)^{\frac{q}{2}} \pi^{\frac{n}{2}}}{4^{k-\frac{n}{2}+1}\left(k-\frac{n}{2}+1\right) !}
$$

and $L^{j}$ is a linear homogeneous diferential operation iterated $\mathrm{j}$ times defined by the following formula

$$
L^{j}=\left\{\frac{\partial^{2}}{\partial x_{1}^{2}}+\ldots \frac{\partial^{2}}{\partial x_{p}^{2}}-\frac{\partial^{2}}{\partial x_{p+1}^{2}}-\cdots-\frac{\partial^{2}}{\partial x_{p+q}^{2}}\right\}^{j} .
$$

The operator $L=\left\{\frac{\partial^{2}}{\partial x_{1}^{2}}+\ldots \frac{\partial^{2}}{\partial x_{p}^{2}}-\frac{\partial^{2}}{\partial x_{p+1}^{2}}-\cdots-\frac{\partial^{2}}{\partial x_{p+q}^{2}}\right\}$ is often called ultahyperbolic.

From [1], page $255,\left(P_{+}^{\lambda}, \varphi\right)$ has two sets of singularities namely

$$
\lambda=-1,-2,-3, \ldots
$$

and

$$
\lambda=-\frac{n}{2},-\frac{n}{2}-1, \ldots
$$

and from [1], pages 256-269 and page 352 we have ([4], page 139, formula (2.27)):

$$
\begin{aligned}
\mathcal{R}\rceil s_{\lambda=-k-1} P_{+}^{\lambda} & =\frac{(-1)^{k}}{k !} \delta_{1}^{(k)}(P) \text { if } p \text { is even and } q \text { odd, } \\
\mathcal{R}\rceil s_{\lambda=-k-1} P_{+}^{\lambda} & =\frac{(-1)^{k}}{k !} \delta_{1}^{(k)}(P) \text { if } p \text { is odd and } q \text { even, } \\
\mathcal{R}\rceil s_{\lambda=-\frac{n}{2}-k} P_{+}^{\lambda} & =0 \text { if } p \text { is even and } q \text { odd }
\end{aligned}
$$

and

$$
\mathcal{R}\rceil s_{\lambda=-\frac{n}{2}-k} P_{+}^{\lambda}=\frac{(-1)^{\frac{q}{2}} \pi^{\frac{n}{2}}}{4^{k} k ! \Gamma\left(\frac{n}{2}+k\right)} L^{k}\{\delta(x\} \text { if } p \text { is odd and } q \text { even. }
$$

where $L^{k}$ is defined by the formula (28).

Similarly $\left(P_{-}^{\lambda}, \varphi\right)$ has singularities in the same points that $\left(P_{+}^{\lambda}, \varphi\right)$ and taking into account all that we have above about $P_{+}^{\lambda}$ remains true also for $P_{-}^{\lambda}$ except that $p$ and $q$ must interchanged, and in all the formulae $\delta_{1}^{(k)}(P)$ must be peplaced by

$$
\delta_{1}^{(k)}(-P)=(-1)^{k} \delta_{2}^{(k)}(P)
$$


and $(L)$ by $(-L)$ (see $([1])$, pages 279 and 352$)$ we have,

$$
\begin{aligned}
\mathcal{R}\rceil s_{\lambda=-k-1} P_{-}^{\lambda} & =\frac{(-1)^{k}}{k !} \delta_{1}^{(k)}(-P) \text { if } p \text { is odd and } q \text { even, } \\
\mathcal{R}\rceil s_{\lambda=-k-1} P_{-}^{\lambda} & =\frac{(-1)^{k}}{k !} \delta_{1}^{(k)}(-P) \text { if } p \text { is even and } q \text { odd, } \\
\mathcal{R}\rceil s_{\lambda=-\frac{n}{2}-k} P_{-}^{\lambda} & =0 \text { if } p \text { is odd and } q \text { even }
\end{aligned}
$$

and

$$
\mathcal{R}\rceil s_{\lambda=-\frac{n}{2}-k} P_{-}^{\lambda}=\frac{(-1)^{\frac{p}{2}} \pi^{\frac{n}{2}}}{4^{k} k ! \Gamma\left(\frac{n}{2}+k\right)}(-L)^{k}\{\delta(x\} \text { if } p \text { is even and } q \text { odd. }
$$

If the dimension $n$ of the space is even and $\mathrm{p}$ and $\mathrm{q}$ are even, $\mathrm{P}_{+}^{\lambda}$ has simple poles at $\lambda=-\frac{n}{2}-k$, where $k$ is non-negative integer, and the residues are given by ([1], p.268 and [4], p.141)

$$
\begin{aligned}
\mathcal{R}\rceil s_{\lambda=-\frac{n}{2}-k, k=0,1,2, . .} P_{+}^{\lambda}= & \frac{(-1)^{\frac{n}{2}+k-1}}{\Gamma\left(\frac{n}{2}+k\right)} \delta_{1}^{\left(\frac{n}{2}+k-1\right)}(P)+ \\
& +\frac{(-1)^{\frac{q}{2}} \pi^{\frac{n}{2}}}{4^{k} k ! \Gamma\left(\frac{n}{2}+k\right)} L^{k}\{\delta(x\}
\end{aligned}
$$

where $L^{k}$ is defined by (28).

If, on the other hand, $p$ and $q$ are odd, $P_{+}^{\lambda}$ has pole of order 2 at $\lambda=-\frac{n}{2}-k$ and from [1], p.269 and [4], p.143, we have

$$
\mathcal{R}\rceil s_{\lambda=-\frac{n}{2}-k} P_{+}^{\lambda}=\frac{(-1)^{\frac{n}{2}+k-1}}{\Gamma\left(\frac{n}{2}+k\right)} \delta_{1}^{\left(\frac{n}{2}+k-1\right)}(P)++\frac{(-1)^{\frac{q+1}{2}} \pi^{\frac{n}{2}-1}}{2^{2 k} k ! \Gamma\left(\frac{n}{2}+k\right)}\left[\psi\left(\frac{p}{2}\right)-\psi\left(\frac{n}{2}\right)\right] \cdot L^{k}\{\delta(x)\},
$$

where

$$
\psi(x)=\frac{\Gamma^{\prime}(x)}{\Gamma(x)} .
$$

and $\Gamma(x)$ is the function gamma defined by

$$
\Gamma(x)=\int_{0}^{\infty} e^{-z} z^{x-1} d z .
$$

([3], Vol.I, p.344).

For integral and half-integral values of the argument, $\psi(x)$ is given by

$$
\begin{aligned}
\psi(k) & =-\gamma+1+\frac{1}{2}+\cdots+\frac{1}{k-1}, \\
\psi\left(k+\frac{1}{2}\right) & =-\gamma-2 \ln (2)+2\left(1+\frac{1}{3}+\cdots+\frac{1}{2 k-1}\right),
\end{aligned}
$$

where $\gamma$ is Euler's constant. 
Similarly

$$
\mathcal{R}\rceil s_{\lambda=-\frac{n}{2}-k} P_{-}^{\lambda}=\frac{(-1)^{\frac{n}{2}+k-1}}{\Gamma\left(\frac{n}{2}+k\right)} \delta_{1}^{\left(\frac{n}{2}+k-1\right)}(-P)++\frac{(-1)^{\frac{p}{2}} \pi^{\frac{n}{2}}}{4^{k} k ! \Gamma\left(\frac{n}{2}+k\right)}(-L)^{k}\{\delta(x\}
$$

if $p$ and $q$ are even, and

$$
\mathcal{R}\rceil s_{\lambda=-\frac{n}{2}-k} P_{-}^{\lambda}=\frac{(-1)^{\frac{n}{2}+k-1}}{\Gamma\left(\frac{n}{2}+k\right)} \delta_{1}^{\left(\frac{n}{2}+k-1\right)}(-P)++\frac{(-) 1^{\frac{p+1}{2}} \pi^{\frac{n}{2}-1}}{2^{2 k} k ! \Gamma\left(\frac{n}{2}+k\right)} \cdot\left[\psi\left(\frac{q}{2}\right)-\psi\left(\frac{n}{2}\right)\right](-L)^{k}\{\delta(x)\}
$$

if $p$ and $q$ are odd

\section{Relations of $k$-th derivative of Dirac delta in hypercone with ultrahyperbolic operator}

In this paragraph we prove that generalized functions $\delta^{(k)}\left(P_{+}\right)-\delta_{1}^{(k)}(P)$ and $\delta^{(k)}\left(P_{-}\right)-$ $\delta_{1}^{(k)}(-P)$ are concentrated in the vertex of the cone $P=0$.

Theorem 1 Let $k$ be non-negative integer and $n$ even dimension of the space then the following formulae are valid,

$$
\delta^{(k)}\left(P_{+}\right)-\delta_{1}^{(k)}(P)=B_{k, p, q} L^{k-\frac{n}{2}+1} \text { if } k \geq \frac{n}{2}-1
$$

where

$$
B_{k, p, q}=\frac{(-1)^{k}(-1)^{\frac{q}{2}} \pi^{\frac{n}{2}}}{4^{k-\frac{n}{2}+1}\left(k-\frac{n}{2}+1\right) !} \text { for } p \text { and } q \text { are both even, }
$$

and

$$
\begin{gathered}
B_{k, p, q}=\frac{(-1)^{k}(-1)^{\frac{q+1}{2}} \pi^{\frac{n}{2}-1}}{4^{k-\frac{n}{2}+1}\left(k-\frac{n}{2}+1\right) !} . \\
{\left[\psi\left(\frac{p}{2}\right)-\psi\left(\frac{n}{2}\right)\right] . L^{k-\frac{n}{2}+1}\{\delta(x)\} \text { for } p \text { and } q \text { are both odd. }}
\end{gathered}
$$

PROOF: From (41),(47) and considering the formulae (19) and (20) under conditions $k \geq$ $\frac{n}{2}-1$,and when $p$ and $q$ are even, we have

$$
\delta^{(k)}\left(P_{+}\right)-\delta_{1}^{(k)}(P)=(-1)^{k} a_{q, n, k} L^{k-\frac{n}{2}+1}\{\delta(x)\} .
$$

where $a_{q, n, k}$ is defined by $(27)$.

Similarly from (42), (48) and considering the formulae (19) and (20) under conditions $k \geq \frac{n}{2}-1$,and when $p$ and $q$ are odd, we have

$$
\begin{gathered}
\delta^{(k)}\left(P_{+}\right)-\delta_{1}^{(k)}(P)=\frac{(-1)^{k}(-1)^{\frac{q+1}{2}} \pi^{\frac{n}{2}-1}}{4^{k-\frac{n}{2}+1}\left(k-\frac{n}{2}+1\right) !} . \\
{\left[\psi\left(\frac{p}{2}\right)-\psi\left(\frac{n}{2}\right)\right] \cdot L^{k-\frac{n}{2}+1}\{\delta(x)\} \text { for p and q are both odd. }}
\end{gathered}
$$


From (52) and (53) we obtain the formula (49),(50) and (51) which proves the theorem.

The formula (49) represent a relation between $\delta^{(k)}\left(P_{+}\right)-\delta_{1}^{(k)}(P)$ and the ultrahyperbolic operator iterated $k-\frac{n}{2}+1$ times under condition $k \geq \frac{n}{2}-1$.

Theorem 2 Let $k$ be non-negative integer and $n$ even dimension of the space, then the following formulae are valid:

$$
\delta^{(k)}\left(P_{-}\right)-\delta_{1}^{(k)}(-P)=D_{k, p, q} L^{k-\frac{n}{2}+1}\{\delta(x)\}
$$

where

$$
D_{k, p, q}=\frac{(-1)(-1)^{\frac{q}{2}} \pi^{\frac{n}{2}}}{4^{k-\frac{n}{2}+1}\left(k-\frac{n}{2}+1\right) !} \text { for } p \text { and } q \text { are both even, }
$$

and

$$
\begin{gathered}
D_{k, p, q}=\frac{(-1)^{\frac{q+1}{2}} \pi^{\frac{n}{2}-1}}{4^{k-\frac{n}{2}+1}\left(k-\frac{n}{2}+1\right) !} \\
{\left[\psi\left(\frac{q}{2}\right)-\psi\left(\frac{n}{2}\right)\right] . L^{k-\frac{n}{2}+1}\{\delta(x)\} \text { for } p \text { and } q \text { are both odd }}
\end{gathered}
$$

PRoOF: From (41),(47) and considering the formulae (19) and (20) under conditions $k \geq$ $\frac{n}{2}-1$,and when $p$ and $q$ are even, we have:

$$
\delta^{(k)}\left(P_{-}\right)-\delta_{1}^{(k)}(-P)=(-1) a_{q, n, k} L^{k-\frac{n}{2}+1}\{\delta(x)\}
$$

where $a_{q, n, k}$ is defined by $(27)$

Similarly from (42), (48) and considering the formulae (19) and (20) under conditions $k \geq \frac{n}{2}-1$, and when $p$ and $q$ are odd, we have:

$$
\begin{gathered}
\delta^{(k)}\left(P_{-}\right)-\delta_{1}^{(k)}(-P)=\frac{(-1)^{\frac{q+1}{2}} \pi^{\frac{n}{2}-1}}{4^{k-\frac{n}{2}+1}\left(k-\frac{n}{2}+1\right) !} \\
{\left[\psi\left(\frac{q}{2}\right)-\psi\left(\frac{n}{2}\right)\right] \cdot L^{k-\frac{n}{2}+1}\{\delta(x)\} \text { for } p \text { and } q \text { are both odd }}
\end{gathered}
$$

From the formulae (57) and (58) we obtain the formulae (54),(55) and (56) which proves the theorem.

The formula (54) represents a relation between $\delta^{(k)}\left(P_{-}\right)-\delta_{1}^{(k)}(-P)$ with the ultrahyperbolic operator iterated $k-\frac{n}{2}+1$ times under condition $k \geq \frac{n}{2}-1$.

Theorem 3 Let $k$ be non-negative integer and $n$ even dimension of the space then the following formulae are valid,

$$
\delta_{1}^{(k)}(P)-\delta_{2}^{(k)}(P)=A_{k, p, q} L^{k-\frac{n}{2}+1}\{\delta(x)\}
$$

where

$$
A_{k, p, q}=\frac{(-1)(-1)^{k}(-1)^{\frac{q}{2}} \pi^{\frac{n}{2}}}{4^{k-\frac{n}{2}+1}\left(k-\frac{n}{2}+1\right) !} \text { for } p \text { and } q \text { are both even, }
$$


and

$$
\begin{gathered}
D_{k, p, q}=\frac{(-1)^{\frac{q+1}{2}} \pi^{\frac{n}{2}-1}}{4^{k-\frac{n}{2}+1}\left(k-\frac{n}{2}+1\right) !} . \\
{\left[\psi\left(\frac{q}{2}\right)-\psi\left(\frac{p}{2}\right)\right] . L^{k-\frac{n}{2}+1}\{\delta(x)\} \text { for } p \text { and } q \text { are both odd }}
\end{gathered}
$$

PROOF: From (49) and (54) using (25), (50) and (60) under conditions $k \geq \frac{n}{2}-1$, and when $p$ and $q$ are even, we have,

$$
\begin{gathered}
\frac{(-1)(-1)^{k}(-1)^{\frac{q}{2}} \pi^{\frac{n}{2}}}{4^{k-\frac{n}{2}+1}\left(k-\frac{n}{2}+1\right) !} L^{k-\frac{n}{2}+1}\{\delta(x)\}=\delta^{(k)}\left(P_{+}\right)-(-1)^{k} \delta^{(k)}\left(P_{-}\right)= \\
\delta_{1}^{(k)}(P)-\delta_{2}^{(k)}(P)+\frac{(-1)^{k}(-1)^{\frac{q}{2}} \pi^{\frac{n}{2}}}{4^{k-\frac{n}{2}+1}\left(k-\frac{n}{2}+1\right) !} L^{k-\frac{n}{2}+1}\{\delta(x)\}+ \\
\frac{(-1)^{k}(-1)^{\frac{q}{2}} \pi^{\frac{n}{2}}}{4^{k-\frac{n}{2}+1}\left(k-\frac{n}{2}+1\right) !} L^{k-\frac{n}{2}+1}\{\delta(x)\}
\end{gathered}
$$

therefore

$$
\delta_{1}^{(k)}(P)-\delta_{2}^{(k)}(P)=\frac{(-1)(-1)^{k}(-1)^{\frac{q}{2}} \pi^{\frac{n}{2}}}{4^{k-\frac{n}{2}+1}\left(k-\frac{n}{2}+1\right) !} L^{k-\frac{n}{2}+1}\{\delta(x)\} .
$$

Similarly from (49) and (54) using (26), (51) and (56) under conditions $k \geq \frac{n}{2}-1$, and when $p$ and $q$ are odd, we have

$$
\begin{gathered}
\delta_{1}^{(k)}(P)-\delta_{2}^{(k)}(P)=\delta^{(k)}\left(P_{+}\right)-(-1)^{k} \delta^{(k)}\left(P_{-}\right)+ \\
+\frac{(-1)^{k}(-1)^{\frac{q+1}{2}} \pi^{\frac{n}{2}-1}}{4^{k-\frac{n}{2}+1}\left(k-\frac{n}{2}+1\right) !} \cdot\left[\psi\left(\frac{n}{2}\right)-\psi\left(\frac{p}{2}\right)+\psi\left(\frac{q}{2}\right)-\psi\left(\frac{n}{2}\right)\right] \cdot L^{k-\frac{n}{2}+1}\{\delta(x)\}= \\
=\frac{(-1)^{k}(-1)^{\frac{q+1}{2}} \pi^{\frac{n}{2}-1}}{4^{k-\frac{n}{2}+1}\left(k-\frac{n}{2}+1\right) !} \cdot\left[\psi\left(\frac{q}{2}\right)-\psi\left(\frac{p}{2}\right)\right] \cdot L^{k-\frac{n}{2}+1}\{\delta(x)\}
\end{gathered}
$$

From the formulae (63) and (64) we obtain the formulae (59), (60) and (61) which proves the theorem.

The formula (59) represent a relation between $\delta_{1}^{(k)}(P)-\delta_{2}^{(k)}(P)$ with the ultrahyperbolic operator iterated $k-\frac{n}{2}+1$ times under condition $k \geq \frac{n}{2}-1$.

\section{References}

[1] Gelfand, I.M.; Shilov, G.E. (1964) Generalized Functions, Vol.I. Academic Press, New York.

[2] Ram Shankar Pathak (1997) Integral Transforms of Generalized Functions and their Applications. Gordon and Breach Science Publishers, Amsterdam. 
[3] Erdelyi, A. (Ed). (1953) Higher Trascendental Functions,Vol. I and II. McGraw-Hill, New York.

[4] Aguirre Téllez, M.A. (1994) The distributional Hankel transform of Marcel Riesz's ultrahyperbolic kernel, Studies in Applied Mathematics 93: 133-162. 